\section{Prevalência de barreiras para a \\ prática de atividade física em \\ adolescentes}

\section{Prevalence of barriers for physical activity in adolescents}

Mariana Silva Santos',"II

Adriano Akira Ferreira Hino, ${ }^{1,11}$

Rodrigo Siqueira Reis ${ }^{1,1,111}$

\section{Ciro Romélio Rodriguez-Añez ${ }^{11, \text { III }}$}

'Programa de Pós-Graduação em Educação Física da Universidade Federal do Paraná - UFPR.

"Curso de Educação Física da Pontifícia Universidade Católica do Paraná PUCPR.

II'Grupo de Pesquisa em atividade física e qualidade de vida.

\section{Resumo}

O objetivo deste estudo foi analisar a prevalência de barreiras e sua associação com a prática de atividades físicas em adolescentes. O presente estudo, transversal, avaliou uma amostra representativa de escolares do ensino médio da rede pública da cidade de Curitiba-PR, Brasil. Um total de 1.609 escolares (59,7\% do gênero feminino) entre 14 e 18 anos de idade reportou, por meio de um questionário, o nível de atividade física e as barreiras para a prática de atividades físicas. Para verificar a associação entre a prevalência de barreiras e a atividade física foi realizada uma análise de regressão logística para cada barreira investigada ajustando para variáveis de confusão (idade e nível socioeconômico). As análises foram realizadas separadamente para meninos e meninas. Apenas $22 \%$ dos meninos e $9 \%$ das meninas atingiram a atual recomendação para atividade física. Entre as 12 barreiras investigadas, apenas "não ter alguém para levar” não diferiu entre os gêneros. Para todas as outras, a percepção de barreiras foi maior entre as meninas $(\mathrm{p}<0,05)$. "Não ter a companhia dos amigos" e "ter preguiça" foram as barreiras mais reportadas pelos meninos $(30,4 \%)$ e meninas $(51,8 \%)$, respectivamente; no entanto a barreira mais fortemente associada com maior prevalência de níveis insuficientes de atividade física foi "preferir fazer outras coisas" para meninos $(\mathrm{OR}=5.02(2,69-9,37) ; \mathrm{p}<0,05) \mathrm{e}$ meninas $(\mathrm{OR}=7.10(3,71-13,60) ; \mathrm{p}<0,05)$. As barreiras percebidas para a prática de atividades físicas foram mais prevalentes entre as meninas e diferiram em grau de importância entre os gêneros.

Palavras-chave: Atividade física. Adolescentes. Prevalência e determinantes epidemiológicos. 


\section{Abstract}

The aim of this study was to analyze the prevalence and association of barriers to physical activity among adolescents. This cross-sectional study evaluated a representative sample of public high school students in Curitiba-PR, Brazil. A total of 1,609 school adolescents (59.7\% male) between 14 and 18 years of age answered a questionnaire on physical activity status and barriers to physical activity. Logistic regressions were conducted for each barrier investigated to verify the association between the prevalence of barriers and physical activity, adjusting for confounding variables (age and socioeconomic status). Analyses were done separately for boys and girls. Only $22 \%$ of boys and $9 \%$ of girls achieved the current physical activity recommendation. Among the 12 barriers investigated, only "there is nobody to take" did not differ between boys and girls. The perception of barriers was higher for girls than boys $(\mathrm{p}<0.05)$ for all other barriers. "Lack of friends company" and "feel lazy" were the barriers most often reported by boys (30.4\%) and girls (51.8\%) respectively; however, the barrier most strongly associated with prevalence of physical inactivity was "prefer to do other things" for both boys $(\mathrm{OR}=5.02(2.69-9.37)$; $\mathrm{p}<0.05)$ and girls $(\mathrm{OR}=7.10(3.71-13.60) ; \mathrm{p}$ $<0.05$ ). Perceived barriers for the practice of physical activity were more prevalent in girls and differed as to the extent of importance between genders.

Keywords: Physical activity. Adolescents. Prevalence and epidemiologic determinants.

\section{Introdução}

Os estudos realizados em crianças e adolescentes têm ganhado especial atenção, principalmente pelo fato de que as principais doenças crônicas não transmissíveis manifestadas durante a idade adulta parecem ter seu início nesses períodos ${ }^{1,2}$. Além disso, fatores de risco para tais doenças, como a inatividade física, parecem ser consolidados nesta fase. De fato, a atividade física na adolescência tem sido estudada como um possível preditor dos níveis de atividade física na idade adulta. Em um estudo representativo sobre tracking da atividade física, realizado no sul do Brasil em 2003, aqueles que relataram a participação em atividades físicas durante a adolescência apresentaram mais chances de serem ativos na idade adulta ${ }^{3}$. Apesar das evidências apontando os benefícios da atividade física para a saúde física e mental ${ }^{4}$, no Brasil a exposição a baixos níveis de atividade física é elevada e parece atingir $39 \%$ a $93,5 \%$ dos adolescentes, dependendo do método de avaliação $0^{5}$.

Uma possível explicação para o baixo nível de atividade física na adolescência é o número de barreiras que dificultam a participação $0^{6}$. As barreiras referem-se a obstáculos percebidos pelo indivíduo que podem reduzir seu engajamento em comportamentos saudáveis? ${ }^{7}$. Segundo Allison et al. ${ }^{6}$, a percepção de barreiras para a atividade física inclui tanto fatores internos (características individuais, menor prioridade para a atividade física, e envolvimento com atividades relacionadas à tecnologia) como fatores externos (influência de alguém ou da família, falta de tempo, inacessibilidade e facilidades de custo).

No estudo de Zunft et al. ${ }^{8}$, composto por 15,239 sujeitos da União Européia, as maiores barreiras para prática de atividades físicas foram "o comprometimento com o trabalho ou o estudo" (28\%) e "a falta do esporte de preferência" (25\%). Em outro estudo, que relacionou as diferenças na percepção de barreiras entre os gêneros, Tergerson e King ${ }^{9}$, estudaram 535 adolescentes 
escolares (245 meninos) e os resultados indicaram que entre as meninas a barreira mais relatada para se exercitar foi a "falta de tempo para o exercício", enquanto para os meninos foi "preferir utilizar o tempo para fazer outras coisas". O mesmo foi observado nas meninas no estudo de Kimm et al. ${ }^{10}$. Das 2,379 participantes $60 \%$ das jovens sedentárias citaram a "falta de tempo" como a principal barreira para a participação em atividades físicas, seguida de "cansaço excessivo" e "a falta de interesse".

Apesar destas evidências, são escassas as informações sobre a as barreiras mais relatadas entre os jovens brasileiros. Até o presente momento foi encontrado apenas um estudo científico sobre o tema na literatura brasileira ${ }^{11}$. De fato, a maioria das evidências disponíveis sobre a prevalência de barreiras para atividade física na população brasileira são oriundas de estudos realizados com adultos ${ }^{12,13}$.

Com escassas evidências na literatura brasileira e as barreiras sendo fortes determinantes na participação de atividades físicas, é importante a realização de estudos com adolescentes que procurem elucidar quais as principais barreiras e a partir destas informações contribuir com recomendações para o aumento de comportamentos fisicamente ativos na determinada população. Portanto, o objetivo deste estudo foi analisar a prevalência de barreiras e sua associação com a prática de atividades físicas em uma amostra representativa de adolescentes.

\section{Métodos}

O presente estudo consiste em uma análise transversal dos dados do projeto "Determinantes da atividade física e obesidade em escolares do ensino médio da rede pública da cidade de Curitiba-PR, Brasil", realizado em 2006. Detalhes da metodologia empregada na coleta e análise de dados estão disponíveis no artigo de Santos et al. ${ }^{14}$.

\section{Participantes}

De acordo com dados da Secretaria
Estadual da Educação do estado do Paraná, no ano de 2006 havia 42.563 alunos matriculados no ensino médio das escolas públicas da cidade de Curitiba. O cálculo amostral foi realizado considerando um erro amostral de $3 \%$, prevalência estimada de $50 \%$ (inatividade física), efeito de desenho de 1,5, excesso de $10 \%$ para a taxa de não resposta e intervalo de confiança de $95 \%$. Portanto, a amostra calculada de acordo com esses critérios foi de 1.609 escolares. Para a análise multivariada foi estabelecida uma razão de prevalência mínima de $1.3(\mathrm{P}=50 \%$; Poder $=$ $80 \%$; IC $=95 \%$ ) sendo necessários ao menos 41 sujeitos por grupo de comparação.

Com o intuito de estabelecer representatividade, foi realizada uma seleção amostral proporcional por conglomerados em dois estágios. No primeiro, estabeleceu-se a proporcionalidade de alunos de acordo com o número de matrículas em cada uma das nove regiões geográficas da cidade, sendo a unidade amostral a escola. Neste estágio, todas as escolas foram consideradas elegíveis e cada região foi considerada um estrato. No segundo estágio, foi determinada a proporcionalidade de alunos em cada uma das três séries do ensino médio ou estratos. As escolas foram selecionadas aleatoriamente de acordo com os estratos à partir de listagem fornecida pela Secretaria Estadual de Educação, de maneira que cada região atendesse a proporção desejada de alunos por série.

\section{Instrumento}

O instrumento para avaliar as barreiras foi desenvolvido pelos pesquisadores, através da técnica de grupos focais ${ }^{14}$. Para tanto, participaram 59 adolescentes (30 meninas - 50,8\%), com idades entre $15 \mathrm{e}$ 18 anos, de quatro instituições de ensino médio da cidade de Curitiba, PR. Foram constituídos oito grupos focais: quatro de meninos (três grupos com sete integrantes cada e um grupo com oito integrantes) e quatro de meninas (dois grupos com oito e dois com sete integrantes cada). Os grupos focais foram realizados em regiões de di- 
ferentes níveis socioeconômicos (NSE) da cidade. Todas as entrevistas estruturadas (grupos focais) foram gravadas, transcritas e após, serem agrupadas de acordo com a classificação sugerida por Sallis e Owen ${ }^{15}$, resultaram em 24 questões.

Estas questões foram aplicadas em outro grupo de adolescentes ( $\mathrm{n}=108 ; 53 \%$ de meninos) para teste da validade fatorial, de construto e fidedignidade (consistência interna e estabilidade temporal). Detalhes dos métodos e resultados das análises são encontrados no artigo de Santos et al. ${ }^{16}$. Das 24 questões aplicadas, 12 emergiram da análise fatorial e apresentaram clareza, correlação com atividade física $(\mathrm{r}=0,19$ a $0,47)$, consistência interna ( $\alpha=0,85$ a 0,86 ) e fidedignidade adequada ( $R=0,46$ a 0,76 ), sendo estas as questões que compuseram o instrumento. As 12 questões finais tinham como opção de resposta uma escala likert de quatro pontos, variando de "discordo muito" até "concordo muito", para cada situação que poderia representar uma barreira para realizar atividades físicas ${ }^{16}$. Para a análise, os adolescentes foram classificados somente de acordo com a presença ou ausência da respectiva barreira percebida.

A atividade física foi reportada pelos adolescentes de acordo com os dias da semana em que foram realizadas atividades físicas de intensidade moderada a vigorosa com duração igual ou superior a 60 minutos seguindo as mesmas padronizações utilizadas pelo Center of Disease Control ${ }^{17}$. Para efeito de análise, os indivíduos foram classificados em "ativos" (quando relataram participar cinco ou mais dias de atividade física moderada a vigorosa por semana) e "insuficientemente ativos" (quando não atingiam tal classificação). A classificação da prática de AF de 60 minutos (AF60) seguiu a recomendação primária para adolescentes ${ }^{18}$.

O índice de massa corporal (IMC) foi obtido pela razão do peso $(\mathrm{kg})$ pela estatura $\left(\mathrm{m}^{2}\right)$, calculado a partir do peso e estatura autoreportados pelos adolescentes. Para a classificação do IMC em sobrepeso e obesidade utilizou-se os pontos de cortes derivados da população brasileira ${ }^{19}$. Desta maneira, os indivíduos foram classificados em "normoponderais" e "excesso de peso" (sobrepeso e/ou obesidade). Mesmo não sendo uma medida objetiva, o peso e a estatura autoreportados demonstram elevada concordância com a avaliação objetiva, podendo ser utilizados para avaliação destas medidas entre adolescentes ${ }^{20,21}$.

Para avaliação do NSE utilizou-se o Critério de Classificação Econômica Brasil ${ }^{22}$. Os indivíduos foram agrupados em três níveis, sendo o nível "A" o maior deles: A (adolescentes de classes A1 e A2), B (adolescentes de classes B1 e B2) e C (adolescentes das classes $\mathrm{C}$, D e E).

\section{Coleta de dados}

A aplicação do questionário aconteceu de maneira coordenada nas salas de aula durante o horário das aulas de educação física. As aplicações foram realizadas por dois entrevistadores previamente treinados para esta finalidade. As entrevistas foram conduzidas após a devida aprovação do comitê de ética em pesquisa (CEP-PUCPR - $\left.\mathrm{n}^{\circ} 1076 / 2006\right)$ e os protocolos seguiram as recomendações do Sistema Nacional de Ética em Pesquisa, que incluem a apresentação do termo de consentimento livre e esclarecido devidamente assinado pelo responsável.

\section{Análise estatística}

Para a descrição da amostra e barreiras relatadas de acordo com o gênero, utilizouse a distribuição de frequências absoluta e relativa, bem como o teste de Qui-quadrado para proporções. Posteriormente, foi testada a associação entre a prática de AF60 e as barreiras para prática de atividade física por meio da regressão logística utilizando dois modelos, analisados separadamente para os gêneros. No primeiro, realizou-se uma regressão bruta assumindo-se AF60 como variável dependente e as barreiras como variável independente. O segundo modelo foi ajustado para as co-variáveis idade e NSE. As análises foram realizadas 
separadamente para o gênero masculino e feminino no software estatístico SPSS 11.0 e o nível de significância mantido em $5 \%$.

\section{Resultados}

Na tabela 1 são apresentas as características da amostra. Participaram deste estudo 1.615 adolescentes (59,69\% meninas), com idades entre 14 e 18 anos. Foi observada maior proporção de adolescentes na faixa dos 16 anos (meninos: 33,18\% e meninas: 33,40\%). Em relação ao NSE, em ambos os gêneros, a maior parte dos adolescentes pertence à classe B. Quanto ao IMC, foi identificada uma maior proporção de adolescentes normoponderais (meninos: $83,57 \%$ e meninas: $91,54 \%$ ). Ao analisar o cumprimento das recomendações de atividade física, identificou-se que uma pequena proporção dos adolescentes de ambos os gêneros atingem a recomendação de AF60, sendo que entre os meninos esta proporção foi de $22,03 \%$, e de $9,06 \%$ entre as meninas. A diferença entre as proporções das variáveis NSE, IMC e AF60, foi significativa do ponto de vista estatístico para o teste de qui-quadrado $(\mathrm{p}<0,05)$.

Entre as 12 barreiras investigadas, apenas "não ter alguém para levar", não diferiu entre os gêneros $(p=0,31)$ (Tabela 2.). Para todas as outras barreiras, a percepção foi maior entre as meninas $(p<0,05)$. Entre meninos e meninas, respectivamente, as barreiras mais prevalentes foram "não ter companhia dos amigos" (30,41\%) e "ter preguiça” (51,76\%). No outro extremo, para ambos, a menos prevalente foi "não consegue realizar os exercícios” (meninos: 8,45\% e meninas: 13,07).

A figura 1 apresenta a distribuição das barreiras de acordo com as recomendações de AF60. Para todas as barreiras investigadas, os adolescentes inativos apresentaram maior prevalência $(\mathrm{p}<0,05)$, em relação aos ativos. As barreiras mais relatadas, entre os inativos, foram "ter preguiça" $(45,83 \%)$, "falta de companhia dos amigos" (43,58\%) e "o clima" (43,29\%). Para os adolescentes ativos, as mais frequentes foram "o clima dificulta" (25,22\%), "falta de companhia dos amigos"

Tabela 1 - Descrição das variáveis sociodemográficas dos participantes do estudo ( $n=1.615)$; adolescentes da rede pública da cidade de ensino Curitiba, PR, 2006.

Table 1 - Socio-demographic variables of study participants $(n=1,615)$; Curitiba public high school students, 2006.

\begin{tabular}{|c|c|c|c|c|c|}
\hline \multirow[t]{2}{*}{ Variáveis } & & \multicolumn{2}{|c|}{ Meninos } & \multicolumn{2}{|c|}{ Meninas } \\
\hline & & f.a. & $\%$ & f.a. & $\%$ \\
\hline Gênero & & 651 & 40,31 & 964 & 59,69 \\
\hline \multirow[t]{5}{*}{ Idade } & 14 & 31 & 4,76 & 57 & 5,91 \\
\hline & 15 & 155 & 23,81 & 254 & 26,35 \\
\hline & 16 & 216 & 33,18 & 322 & 33,40 \\
\hline & 17 & 190 & 29,19 & 254 & 26,35 \\
\hline & 18 & 59 & 9,06 & 77 & 7,99 \\
\hline \multirow[t]{3}{*}{ NSE* } & A & 103 & 15,82 & 199 & 20,64 \\
\hline & B & 407 & 62,52 & 603 & 62,55 \\
\hline & C & 141 & 21,66 & 162 & 16,80 \\
\hline \multirow[t]{2}{*}{$\mathrm{IMC}^{*}$} & Normoponderais & 519 & 83,57 & 833 & 91,54 \\
\hline & Excesso de peso & 102 & 16,43 & 77 & 8,46 \\
\hline \multirow[t]{2}{*}{ AF60* } & Ativos & 143 & 22,03 & 87 & 9,06 \\
\hline & Inativos & 506 & 77,97 & 873 & 90,94 \\
\hline
\end{tabular}

f.a: frequência absoluta, ${ }^{*} \mathrm{p}<0,05$ para o teste de Qui-quadrado (Comparação entre os gêneros). f.a: absolute frequency, ${ }^{*} p<0.05$ for chi-square test (Comparison between genders). 
Tabela 2 - Presença de barreiras para a prática de atividade física de acordo com o gênero.

Table 2 - Presence of barriers to physical activity according to gender.

\begin{tabular}{|c|c|c|c|c|c|c|}
\hline \multirow[t]{2}{*}{ Barreiras } & \multicolumn{2}{|c|}{ Meninos } & \multicolumn{2}{|c|}{ Meninas } & \multirow[t]{2}{*}{$x^{2}$} & \multirow[t]{2}{*}{$\mathrm{p}$} \\
\hline & f.a. & $\%$ & f.a & $\%$ & & \\
\hline 1. Não conhece muito bem os locais & 189 & 29,03 & 345 & 35,79 & 8,0 & 0,001 \\
\hline 2. Não tem alguém para levar & 197 & 30,26 & 315 & 32,68 & 1,0 & 0,31 \\
\hline 3. Não consegue realizar os exercícios & 55 & 8,45 & 126 & 13,07 & 8,3 & 0,001 \\
\hline 4. Prefere fazer outras coisas & 169 & 25,96 & 451 & 46,78 & 71,2 & $<0,001$ \\
\hline 5. Não tem motivação & 116 & 17,82 & 291 & 30,19 & 31,5 & $<0,001$ \\
\hline 6. Tem preguiça & 173 & 26,57 & 499 & 51,76 & 101,5 & $<0,001$ \\
\hline 7. Não tem companhia dos amigos & 198 & 30,41 & 461 & 47,82 & 48,7 & $<0,001$ \\
\hline 8. Acha chato & 96 & 14,75 & 254 & 26,35 & 30,8 & $<0,001$ \\
\hline 9. Ninguém o convida & 151 & 23,20 & 274 & 28,42 & 5,5 & 0,02 \\
\hline 10. Não tem tempo & 152 & 23,35 & 312 & 32,37 & 15,4 & $<0,001$ \\
\hline 11. O clima dificulta & 186 & 28,57 & 472 & 48,96 & 66,9 & $<0,001$ \\
\hline 12. Não tem como pagar & 83 & 12,75 & 215 & 22,30 & 23,6 & $<0,001$ \\
\hline
\end{tabular}

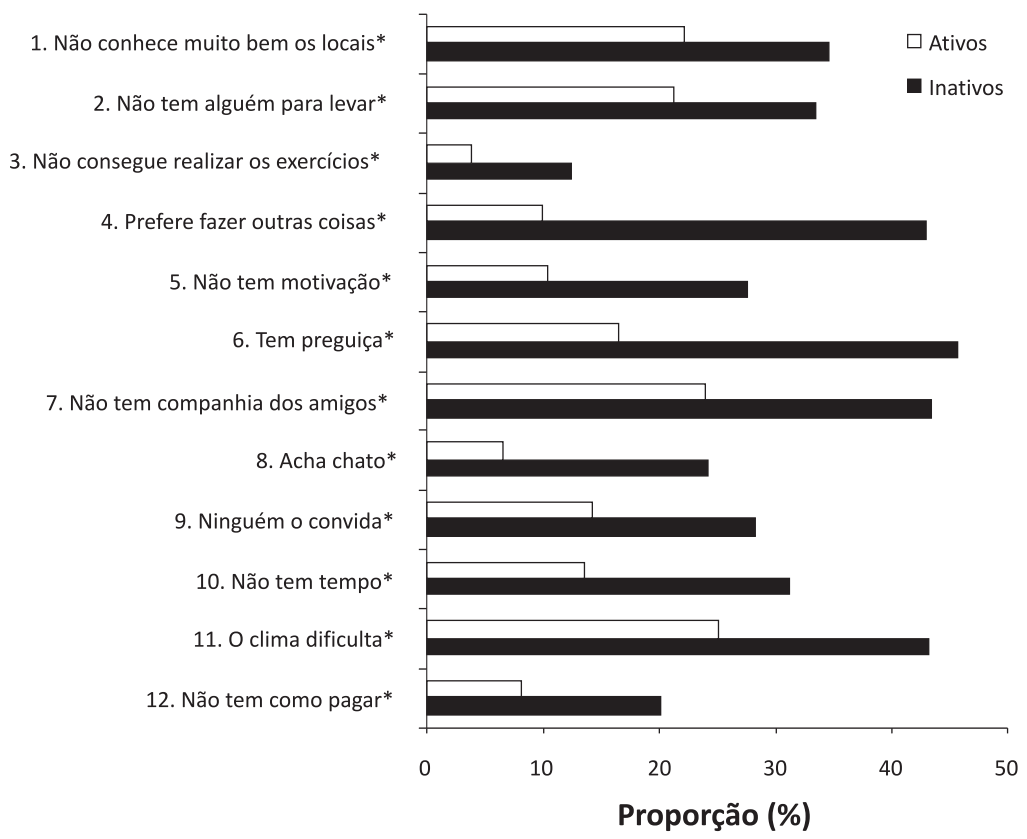

${ }^{*} p<0,05$ para o teste Qui-quadrado. ${ }^{*} p<0,005$ for the chi-square test.

Figura 1 - Prevalência de barreiras percebidas de acordo com a recomendação de atividade física (AF60).

Figure 1 - Prevalence of perceived barriers according to the physical activity recommendation.

(23,91\%) e "não conhecimento dos locais"

$(22,17 \%)$. Tanto entre os adolescentes ativos quanto para os inativos, a barreira menos relatada foi "Não consegue realizar os exercícios” (12,4\% e 3,91\%, respectivamente).
Quando observados os resultados entre os meninos (Tabela 3), as barreiras "não conhece muito bem os locais", "não tem alguém para levar ou buscar" e "não consegue realizar os exercícios" não foram associadas 
Tabela 3 - Odds ratio (OR) de inatividade física de acordo com a presença de barreiras percebidas.

Table 3 - Odds ratio (OR) to physical inactivity according to presence of perceived barriers.

\begin{tabular}{|c|c|c|c|c|c|c|c|c|}
\hline \multirow[t]{2}{*}{ Barreiras } & \multicolumn{4}{|c|}{ Meninos } & \multicolumn{4}{|c|}{ Meninas } \\
\hline & $\begin{array}{l}\text { OR } \\
\text { Bruto }\end{array}$ & $\left(\mathrm{IC}_{95 \%}\right)$ & $\begin{array}{c}\text { OR } \\
\text { Ajustado }\end{array}$ & $\left(\mathrm{IC}_{95 \%}\right)$ & $\begin{array}{l}\text { OR } \\
\text { Bruto }\end{array}$ & $\left(\mathrm{IC}_{95 \%}\right)$ & $\begin{array}{c}\text { OR } \\
\text { Ajustado }\end{array}$ & $\left(\mathrm{IC} \mathrm{95 \%}_{95}\right)$ \\
\hline $\begin{array}{l}\text { 1. Não conhece muito bem os } \\
\text { locais }\end{array}$ & 1,42 & $(0,91-2,15)$ & 1,33 & $(0,87-2,06)$ & 2,44 & $(1,41-4,22)$ & 2,33 & $(1,34-4,05)$ \\
\hline 2. Não tem alguém para levar & 1,58 & $(1,30-2,44)$ & 1,48 & $(0,95-2,30)$ & 2,30 & $(1,31-4,02)$ & 2,23 & $(1,27-3,92)$ \\
\hline $\begin{array}{l}\text { 3. Não consegue realizar os } \\
\text { exercícios }\end{array}$ & 1,99 & $(0,88-4,50)$ & 2,02 & $(0,89-4,60)$ & 7,01 & $\begin{array}{l}(1,70- \\
28,77)\end{array}$ & 7,01 & $(1,71-28,84)$ \\
\hline 4. Prefere fazer outras coisas & 4,91 & $(2,64-9,31)$ & 5,02 & $(2,69-9,37)$ & 6,92 & $\begin{array}{l}(3,63- \\
13,21)\end{array}$ & 7,10 & $(3,71-13,60)$ \\
\hline 5. Não tem motivação & 2,52 & $(1,37-4,65)$ & 2,51 & $(1,36-4,64)$ & 3,24 & $(1,70-6,20)$ & 3,31 & $(1,73-6,35)$ \\
\hline 6. Tem preguiça & 3,84 & $(2,18-6,77)$ & 3,97 & $(2,24-7,03)$ & 3,32 & $(2,02-5,45)$ & 3,39 & $(2,06-5,58)$ \\
\hline $\begin{array}{l}\text { 7. Não tem companhia dos } \\
\text { amigos }\end{array}$ & 2,58 & $(1,60-4,14)$ & 2,53 & $(1,56-4,80)$ & 1,74 & $(1,10-2,74)$ & 1,75 & $(1,10-2,77)$ \\
\hline 8. Acha chato & 3,55 & $(1,68-7,51)$ & 3,63 & $(1,71-7,71)$ & 4,48 & $(2,04-9,84)$ & 4,68 & $(2,12-10,33)$ \\
\hline 9. Ninguém o convida & 2,28 & $(1,35-3,84)$ & 2,23 & $(1,32-3,77)$ & 2,20 & $(1,22-3,96)$ & 2,13 & $(1,18-3,85)$ \\
\hline 10. Não tem tempo & 3,17 & $(1,79-5,60)$ & 3,17 & $(1,78-5,63)$ & 2,25 & $(1,28-3,94)$ & 2,27 & $(1,29-4,00)$ \\
\hline 11. O clima dificulta & 2,20 & $(1,37-3,52)$ & 2,16 & $(1,35-3,47)$ & 1,63 & $(1,04-2,57)$ & 1,63 & $(0,13-2,57)$ \\
\hline 12. Não tem como pagar & 4,10 & $(1,74-9,61)$ & 3,95 & $(1,68-9,31)$ & 1,70 & $(0,92-3,13)$ & 1,59 & $(0,86-2,94)$ \\
\hline
\end{tabular}

Ajuste: idade e NSE

com a inatividade física. Entre as meninas as barreiras que não foram associadas foram as barreiras que não apresentaram associação com inatividade física: "não tem como pagar" e "o clima dificulta".

Na análise ajustada para idade e nível socioeconômico, as maiores chances para apresentar inatividade física foram encontradas entre os adolescentes que relataram perceber a barreira "preferir fazer outras coisas" (meninos: 5,02 e meninas: 7,10). As barreiras que não apresentaram associação significativa na análise bruta também não foram associadas na análise ajustada.

\section{Discussão}

O presente estudo analisou a prevalência de barreiras relatadas em uma amostra representativa de adolescentes da cidade de Curitiba, PR. Em geral, as meninas e adolescentes fisicamente inativos relataram mais barreiras do que meninos e adolescentes fisicamente ativos, respectivamente. As barreiras mais relatadas não foram aquelas que apresentaram associação com a inatividade física.

Muitos estudos têm identificado um decréscimo nos níveis de atividade física da população em geral, o que é uma fonte de preocupação, uma vez que um estilo de vida inativo pode contribuir para o desenvolvimento de muitas doenças, em especial a obesidade. O presente estudo, realizado em uma amostra representativa de adolescentes escolarizados da rede pública da cidade de Curitiba, aponta que os níveis de atividade física apresentados pelos adolescentes são bastante preocupantes, principalmente entre as meninas. Apenas $22 \%$ dos meninos e 9,1\% das meninas atingiram a recomendação de atividades físicas necessárias para atingir benefícios em sua saúde.

De maneira geral os meninos tendem a possuir maiores níveis de atividade física quando comparados com as meninas ${ }^{23,24}$, já existindo algum consenso na literatura de que esta diferença pode ser explicada por fatores socioculturais ${ }^{25-27}$. Desde as idades mais precoces já é concebida a idéia de 
que homens e mulheres possuem papéis diferentes na sociedade, sendo os homens mais tradicionalmente caracterizados por tarefas voltadas ao trabalho e atividades manuais enquanto as mulheres são caracterizadas para atividades familiares e tarefas domésticas ${ }^{25,26}$. Esta diferença nos níveis de atividade física pode ainda ser maior quando o instrumento utilizado para avaliar a atividade física não contempla atividades ocupacionais, tarefas domésticas, transporte ou atividades familiares ${ }^{28}$. No entanto, mesmo quando são utilizados métodos objetivos para avaliar atividade física, os meninos são mais fisicamente ativos do que as meninas ${ }^{29}$.

No presente estudo as barreiras "falta de companhia dos amigos" e "não ter alguém para levar", obtiveram a maior frequência de relatos entre os meninos (>30\%). Esse resultado revela que o apoio social exerce importante influência no comportamento fisicamente ativo dos meninos. Ainda que a família seja uma das instituições que mais exerce influência no envolvimento de crianças e adolescentes em comportamentos saudáveis, na adolescência os jovens tendem a "ignorar" os conselhos, os valores e as atitudes dos seus pais. Isso ocorre em virtude do desenvolvimento do senso de autonomia, independência e afirmação que faz com que o adolescente dê mais relevância aos hábitos e comportamentos dos seus pares ou de outros agentes socialmente relevantes ${ }^{30}$. Allison et al. ${ }^{6}$ identificaram a ausência de apoio para prática de atividade física da família e dos amigos como importantes barreiras entre meninos.

Em um estudo de revisão, considerando o período dos 10 aos 18 anos, Seabra et al. ${ }^{30}$, relataram que há evidências de que jovens mais ativos tem amigos igualmente ativos. King, Tergerson e Wilson ${ }^{31}$ identificaram que adolescentes que têm a companhia de um amigo para realizar exercícios se engajam significativamente em mais dias de atividade física de intensidade moderada e vigorosa do que os adolescentes que não têm. Desta forma, as intervenções devem direcionar suas ações não somente para o sujeito, mas também para a rede social no entorno deste, aumentando assim as chances de maior efetividade no aumento dos níveis de atividade física.

Com exceção apenas da barreira "não ter alguém para levar", todas as restantes foram mais prevalentes entre as meninas. $\mathrm{O}$ mesmo resultado foi observado no estudo deWu et al. ${ }^{32}$ com adolescentes tailandeses, no entanto, nesse estudo a percepção de barreiras não foi associada com a atividade física. Estudos realizados recentemente encontraram que a principal barreira para a atividade física em meninas é a falta de tempo ${ }^{9,10,33}$. Entretanto, Kimm et al. ${ }^{10}$, observaram que as meninas que relatam esta barreira não passam mais tempo trabalhando ou envolvidas em afazeres domésticos quando comparadas com as que não relatam. Esse resultado indica que esta barreira é um fator mais subjetivo do que uma real causa externa ${ }^{10}$. No atual estudo, a barreira mais prevalente entre as meninas, foi "preguiça" (51,76\% dos relatos) e esse sim parece ser o real motivo para os baixos níveis de atividade física entre as adolescentes. A falta de tempo ocupou somente o sétimo lugar em ordem de importância, com 32,4\% dos relatos.

A maior prevalência de barreiras, quando observada a classificação de atividade física, foi verificada entre os adolescentes inativos. As principais barreiras relatadas para este grupo foram a "preguiça" e a "falta de companhia dos amigos". Esse resultado reforça mais uma vez o apoio social como um importante determinante da prática de atividade física. A terceira barreira mais frequente entre os adolescentes inativos foi "o clima dificulta". Muito embora o presente estudo avalie a percepção desta barreira, pesquisas utilizando medidas objetivas têm evidenciado que o clima parece realmente influenciar no comportamento fisicamente ativos das pessoas, confirmando os achados do presente estudo ${ }^{34,35}$.

Tomando como base a classificação proposta por Sallis e Owen ${ }^{15}$, as barreiras identificadas como tendo maior prevalência entre os adolescentes inativos do presente 
estudo são de ordem psicológica, cognitiva e emocional (preguiça), social e cultural (falta de companhia dos amigos) e ambiental (clima). Entre os adolescentes suficientemente ativos as barreiras são identificadas como ambientais (clima) e sociais e culturais (falta de companhia dos amigos e não conhecimento dos locais). Essa diversidade de dimensões observadas na associação entre a AF60 e as barreiras para a prática de atividades físicas são observadas em diversos modelos e teorias, reforçando o fato de que não existe uma única variável de influência, uma vez que o comportamento ativo é complexo e multifatorial.

As barreiras mais relatadas entre os meninos não foram as mais fortemente associadas com a inatividade física. De fato, "preferir fazer outras coisas", "ter preguiça" e não "ter como pagar" foram as três barreiras mais fortemente associadas entre os meninos. Similarmente o estudo realizado por Brown ${ }^{36}$ com adolescentes americanos também demonstrou que a barreira mais fortemente associada com a atividade física entre adolescentes de ambos os gêneros foi a baixa motivação $(r=-54 ; p<0,01)$ seguida por falta de tempo $(r=-38 ; p<0,01)$.

A grande maioria das observações a respeito das barreiras é obtida através de estudos com delineamento transversal. Em um levantamento longitudinal de três anos realizado por Kimm et al. ${ }^{10} \mathrm{com} 2,379$ meninas, foi observado que entre as 10 barreiras investigadas, "preferir fazer outras coisas ao invés de se exercitar” foi a única preditora de menores níveis de atividade física, indicando que as barreiras de caráter individual ou pessoais parecem ser mais consistentemente associadas com menores níveis de atividade física entre adolescentes. Outro estudo acompanhou meninas adolescentes com risco de comportamentos sedentários e sobrepeso e identificou que os dois fatores mais consistentemente associados com mudanças na atividade física foram ausência de tempo e suporte de pessoas para a prática de atividade física ${ }^{37}$.

Apesar dos estudos citados investigarem barreiras específicas, revisões de literatura realizadas em $2000^{23}$ e $2007^{24}$ avaliaram a percepção de barreiras como um todo em estudos realizados entre 1970 e 2006 e concluíram que entre adolescentes de ambos os gêneros a associação entre as barreiras percebidas e nível de atividade física é inexistente ${ }^{23}$ ou inconclusiva ${ }^{24}$.Contudo, quando são analisados estudos que testaram a associação entre barreiras e atividade física somente entre meninas os resultados são mais consistentes, demonstrando existir uma associação de magnitude pequena para moderada ${ }^{38}$.

Os resultados do presente estudo oferecem direcionamento para que profissionais de saúde e gestores de políticas de promoção da atividade física possam desenvolver estratégias de intervenção direcionadas às barreiras mais frequentes entre os jovens e, portanto, com maior chance de efetividade. Entre os meninos parece que as intervenções devem incluir atividades que favoreçam o aumento das relações sociais, e entre as meninas é fundamental que as intervenções considerem a dificuldade encontrada para realizar os movimentos. Os resultados demonstram que é muito importante que as intervenções sejam atrativas o suficiente para superar a preferência em fazer outras coisas e o fato de achar a atividade física "chata". Entretanto, algumas questões não puderam ser mais bem exploradas no escopo do presente estudo, como a existência de reais barreiras para a atividade física e não apenas aquelas percebidas, o que pode ser melhor explorado em estudos com delineamento mais experimental.

Dentre as limitações do atual estudo, deve-se ressaltar que o delineamento transversal adotado não permite identificar relações de causalidade entre as variáveis, apesar de alguns estudos de acompanhamento já terem confirmado qual o sentido desta relação ${ }^{10}$. Outro ponto importante a ser considerado é que as barreiras foram autoreportadas e, portanto, aspectos que se apresentem como motivos reais podem se confundir com possíveis "desculpas" relacionadas à prática de atividade físicas. No entanto, outras limitações potenciais 
foram melhor controladas. As variáveis de confusão mais comumente associadas à atividade física foram incluídas nos modelos de análise (ex: sexo, idade, classe social e IMC). Embora a amostra tenha sido suficientemente robusta para as análises de prevalência, não foram incluídos estudantes da rede privada de ensino, o que limita os achados àqueles jovens matriculados na rede pública.

No entanto, deve-se considerar que o instrumento desenvolvido para o estudo seguiu um processo rigoroso de desenvolvimento e teve como origem do seu conteúdo relatos e situações identificadas por elementos da população do estudo o que garante maior proximidade das questões com o contexto. Outros aspectos positivos referem-se à representatividade e ao tamanho da amostra, que permitem maior segurança tanto na extrapolação dos resultados para a população alvo, quanto no poder das análises.

\section{Conclusão}

Considerando as limitações acima citadas, conclui-se que existe uma elevada prevalência de adolescentes inativos na cidade de Curitiba, PR, indicando a necessidade de intervenções para esta população em específico.

As barreiras percebidas para a prática de atividade física foram mais prevalentes entre as meninas e também diferiram em grau de importância entre os gêneros, indicando que entre os meninos as barreiras de caráter social são mais predominantes, enquanto que entre as meninas aparecem em destaque as cognitivas e ambientais. No entanto, as barreias mais prevalentes não foram as mais fortemente associadas com a atividade física.

\section{Referências}

1. Telama R, Yang X, Laakso L, Viikari J. Physical activity in childhood and adolescence as predictor of physical activity in young adulthood. Am J Prev Med 1997;13(4):317-23.

2. Kidd P, Reed D, Weaver L, Westneat S, Rayens MK. The transtheoretical model of change in adolescents: implications for injury prevention. J Safety Res 2003;34(3):281-8.

3. Azevedo MR, Araujo CL, Cozzensa da Silva M, Hallal PC. Tracking of physical activity from adolescence to adulthood: a population-based study. Rev Saude Publica 2007;41(1):69-75.

4. Strong WB, Malina RM, Blimkie CJ, Daniels SR, Dishman RK, Gutin B, et al. Evidence based physical activity for school-age youth. J Pediatr 2005;146(6):732-737.

5. Tassitano RM, Bezerra J, Tenório MCM, Colares V, Barros MVG, Hallal PC. Atividade física em adolescentes brasileiros: uma revisão sistemática. Rev Bras Cine Des Hum 2007;9(1):55-60.

6. Allison KR, Dwyer JJ, Goldenberg E, Fein A, Yoshida KK, Boutilier M. Male adolescents' reasons for participating in physical activity, barriers to participation, and suggestions for increasing participation. Adolescence 2005;40(157):155-70.

7. Brown SA. Measuring perceived benefits and perceived barriers for physical activity. Am J Health Behav 2005;29(2):107-16.
8. Zunft HJ, Friebe D, Seppelt B, Widhalm K, Remaut de Winter AM, Vaz de Almeida MD, et al. Perceived benefits and barriers to physical activity in a nationally representative sample in the European Union. Public Health Nutr 1999;2(1A):153-60.

9. Tergerson JL, King KA. Do perceived cues, benefits, and barriers to physical activity differ between male and female adolescents? J Sch Health 2002;72(9):374-80.

10. Kimm SY, Glynn NW, McMahon RP, Voorhees CC, Striegel-Moore RH, Daniels SR. Self-perceived barriers to activity participation among sedentary adolescent girls. Med Sci Sports Exerc 2006;38(3):534-40.

11. Ceschini F, Figueira JA. Barreiras e determinantes para a prática de atividade física em adolescentes. $\mathrm{R}$ bras Ci e Mov 2007;15(1):29-36.

12. Reichert FF, Barros AJ, Domingues MR, Hallal PC. The role of perceived personal barriers to engagement in leisure-time physical activity. Am J Public Health 2007;97(3):515-9.

13. Gobbi S, Caritá LP, Hirayama MS, Quadros-Junior ACd, Santos RF, Gobbi LTB. Comportamento e Barreiras: Atividade Física em Idosos Institucionalizados. Psicologia: Teoria e Pesquisa 2008;24(4):451-458.

14. Santos MS, Fermino RC, Reis RS, Cassou AC, RodriguezAñez CR. Barreiras para a prática de atividade física em adolescentes. Um estudo por grupos focais. Rev Bras Cineantrop Desempenho Hum 2010. 
15. Sallis JF, Owen N. Physical activity \& behavioral medicine. Thousand Oaks, California: SAGE; 1999.

16. Santos MS, Reis RS, Rodriguez-Añez CR, Fermino RC. Desenvolvimento de um instrumento para avaliar barreiras para a prática de atividade física em adolescentes. Revista Brasileira de Atividade Física \& Saúde 2009;14(2).

17. Brener ND, Kann L, Kinchen SA, Grunbaum JA, Whalen L, Eaton D, et al. Methodology of the youth risk behavior surveillance system. MMWR Recomm Rep 2004;53(RR12):1-13.

18. Sallis JF, Patrick K. Physical Activity Guidelines for Adolescents: Consensus Statement. Pediatr Exerc Sci 1994;6:302-314.

19. Conde WL, Monteiro CA. Body mass index cutoff points for evaluation of nutritional status in Brazilian children and adolescents. J Pediatr (Rio J) 2006;82(4):266-72.

20. Farias Júnior JC. Validade das medidas auto-referidas de peso e estatura para o diagnóstico do estado nutricional de adolescentes. Rev. Bras. Saúde Matern. Infant 2007;7(2):167-174.

21. Goodman E, Hinden BR, Khandelwal S. Accuracy of teen and parental reports of obesity and body mass index. Pediatrics 2000;106(1 Pt 1):52-8.

22. ANEP. Brazilian Criteria for Economic Classification. In: Brasília: Brazilian National Association of Research Institutes; 2002.

23. Sallis JF, Prochaska JJ, Taylor WC. A review of correlates of physical activity of children and adolescents. Med Sci Sports Exerc 2000;32(5):963-975.

24. Van Der Horst K, Paw MJ, Twisk JW, Van Mechelen W. A brief review on correlates of physical activity and sedentariness in youth. Med Sci Sports Exerc 2007;39(8):1241-50.

25. Teixeira e Seabra AF, Maia JA, Mendonca DM, Thomis M, Caspersen CJ, Fulton JE. Age and sex differences in physical activity of Portuguese adolescents. Med Sci Sports Exerc 2008;40(1):65-70.

26. Wold B, Hendry L. Social and environmental factors associated with physical activity in young people In: Biddle S, Sallis J, Cavill N, editors. Young and Active? Young people and health-enhancing physical activity evidence and implications. London: Health Education Authority; 1998. p. 176.

27. Li M, Dibley MJ, Sibbritt D, Yan H. Factors associated with adolescents' physical inactivity in Xi' an City, China. Med Sci Sports Exerc 2006;38(12):2075-85.
28. Ainsworth BE. Issues in the assessment of physical activity in women. Res Q Exerc Sport 2000;71(2 Suppl):S37-42.

29. Troiano RP, Berrigan D, Dodd KW, Masse LC, Tilert T, McDowell M. Physical activity in the United States measured by accelerometer. Med Sci Sports Exerc 2008;40(1):181-8.

30. Seabra AF, Mendonca DM, Thomis MA, Anjos LA, Maia JA. [Biological and socio-cultural determinants of physical activity in adolescents]. Cad Saude Publica 2008;24(4):721-36.

31. King KA, Tergerson JL, Wilson BR. Effect of social support on adolescents' perceptions of and engagement in physical activity. J Phys Act Health 2008;5(3):374-84.

32. Wu SY, Pender N, Noureddine S. Gender differences in the psychosocial and cognitive correlates of physical activity among Taiwanese adolescents: a structural equation modeling approach. Int J Behav Med 2003;10(2):93-105.

33. Dwyer JJ, Allison KR, Goldenberg ER, Fein AJ, Yoshida KK, Boutilier MA. Adolescent girls‘ perceived barriers to participation in physical activity. Adolescence 2006;41(161):75-89.

34. Duncan JS, Hopkins WG, Schofield G, Duncan EK. Effects of weather on pedometer-determined physical activity in children. Med Sci Sports Exerc 2008;40(8):1432-8.

35. Chan CB, Ryan DA, Tudor-Locke C. Relationship between objective measures of physical activity and weather: a longitudinal study. Int J Behav Nutr Phys Act 2006;3:21.

36. Brown SA, Huber D, Bergman A. A perceived Benefits and Barriers Scale for strenuous physical activity in college students. Am J Health Promot 2006;21(2):137-40.

37. Neumark-Sztainer D, Story M, Hannan PJ, Tharp T, Rex J. Factors associated with changes in physical activity: a cohort study of inactive adolescent girls. Arch Pediatr Adolesc Med 2003;157(8):803-10.

38. Biddle SJH, Whitehead SH, O’Donovan TM, Nevill ME. Correlates of Participation in Physical Activity for Adolescent Girls: A Systematic Review of Recent Literature. J Phys Act Health 2005;2(4):423-434.

Recebido em: 17/04/09 Versão final reapresentada em: 30/09/09 Aprovado em: 17/11/09 